\title{
Lung Cancer Detection using Curvelet Transform and Neural Network
}

\author{
Bhawna Gupta \\ M.tech (CSE) \\ MITS,Laxmangarh
}

\author{
Shamik Tiwari \\ Assistant Professor \\ MITS, Laxmangarh
}

\begin{abstract}
Throughout the world the common cause of death in humans is lung cancer. It is necessary to detect cancer as early as possible to increase the survival rate. Lung cancer in CT scan images can be classified easily and efficiently using digital image processing techniques. Curvelet transform can extract the features of lung cancer CT scan images proficiently. All extracted feature by curvelet transform are applied to the neural network for training and testing. The performance of proposed work show efficient results.
\end{abstract}

\section{Keywords}

Lung cancer, Curvelet transforms, Neural Network.

\section{INTRODUCTION}

Lung cancer is the independent growth of aberrant cells in one or both lungs. It is the number one cause of deaths in humans. Survival from lung cancer is proportional to its detection time. If it is detected in early stages it increases the rate of survival. According to the latest statistics, a total of 1,660,290 new cancer cases and 580,350 deaths from cancer are projected to occur in the US in 2013. Cancer that starts in the lung is known as primary cancer. The main types of lung cancer are small-cell lung carcinoma (SCLC), also called oat cell cancer, and non-small-cell lung carcinoma (NSCLC). The most common symptoms are coughing (including coughing up blood), weight loss and shortness of breath. Chest Radiograph (x-ray), Computed Tomography (CT), Magnetic Resonance Imaging (MRI scan) and Sputum Cytology are the techniques to diagnose the lung cancer. In terms of speed, analysis of large volume of data CT images has more advantage over the other techniques [1].

Image processing techniques provide an efficient tool to classify the cancer from the CT images. In medical image processing, many medical images do not exhibit regions of uniform and smooth intensities. A huge amount of texture information relevant to clinical practice is acquired by medical images. Texture analysis is widely used for the detection of lung cancer. Neural network is also used for the detection of the lung cancer. Various works have been done in the detection of lung cancer using texture feature extraction and the combination of neural network. Patil et al. [2] have proposed a technique in which Gray Level Co-occurrence Matrix technique is used for texture feature. Yang Yu et al. [3] designed an enhancement filter as preprocessing step. Nodular texture is used for the extraction and, contrast limiting adaptive histogram equalization method is used for the enhancement of the ROI (Region of Interest). Takemura [4] proposed a novel approach to the detection and enhancement of the lung nodule. This recommended method is a two-step. Firstly, a 2D multiscale filtering is used for the detection of lung nodule and then false-positive nodules are reduced by logical AND operator of continuous CT slices. In our research we used curvelet transform to extract the feature of CT scan image and according to these features we train the neural network to get the proficient result.

\subsection{Curvelet Transform}

For multi-resolution, analysis of images wavelets provides a powerful tool. Wavelet based feature are used in many application like image denoising, image compression and tumor recognition. For piecewise, smooth functions in one dimension wavelets provide good performance, but wavelet is not suitable to capture more directional features in an image. To overcome the limitations of wavelet transform Candes and et al. [5] developed curvelet for providing efficient representation of smooth objects with discontinuities along curves in 2000. In curvelet transform basis function are needle shapes and have high directional sensitivity and anisotropy. It was designed to represent edges and other singularities along curves much more efficiently than traditional transforms. Curvelet transform has binary square window, which means subdividing a curve into approximate straight to carry out ridge transform [6]. Curvelet transform can be defined by a pair of windows $\mathrm{W}(\mathrm{r})$ (a radial window) and $\mathrm{V}(\mathrm{t})$ (an angular window), with variables $\mathrm{W}$ as a frequency-domain variable, and $\mathrm{r}$ and $\theta$ as polar coordinates in the frequency-domain

$\sum_{j=-\infty}^{\infty} W^{2}\left(2^{j} r\right)=1, r \in\left(\frac{3}{4}, \frac{3}{2}\right)$

$\sum_{l=-\infty}^{\infty} V^{2}(t-l)=1, t \in\left(-\frac{1}{2}, \frac{1}{2}\right)$

A polar 'wedge' represented by $\mathrm{U}_{\mathrm{j}}$ is supported by $\mathrm{W}$ and $\mathrm{V}$, the radial and angular windows. $\mathrm{U}_{\mathrm{j}}$ defined in Fourier domain by

$U_{j}(r, \theta)=2^{-(3 j / 4)} W\left(2^{-j} r\right) V\left(\frac{2^{-j / 2} \theta}{2 \Pi}\right)$

The curvelet transform can be defined as a function of $\mathrm{x}=(\mathrm{x} 1$, $\mathrm{x} 2$ ) at scale $2^{-\mathrm{j}}$, orientation $\theta$ and position $\mathrm{x}_{\mathrm{k}}^{\mathrm{j}, \mathrm{l}}$ by

$\varphi_{\mathrm{j}, \mathrm{l}, \mathrm{k}}(\mathrm{x})=\varphi_{\mathrm{j}}\left(\mathrm{R}_{\theta_{\mathrm{l}}}\left(\mathrm{x}-\mathrm{x}_{\mathrm{k}}^{(\mathrm{j}, \mathrm{l})}\right)\right)$

Where $R_{\theta}$ is the rotation in radians

Curvelet transform is implemented using fast discrete curvelet transform. The complete process of feature extraction follows these steps [7].

(i) Both image and curvelet filter bank are transformed into Fourier domain.

(ii) Then the convolution of the curvelet with the image in spatial domain becomes the product in Fourier domain.

(iii) Finally to compute the curvelet coefficient inverse Fourier transform is applied. But due the frequency response of curvelet is non rectangular wedge, the wedge 
must be wrapped into rectangular to perform inverse Fourier transform.

(iv) By periodic wedge tiling wrapping is done, then collecting the rectangular coefficient area in the centre.

After that inverse Fourier transform is applied and curvelet coefficients are obtained. The whole process is shown in figure 1.

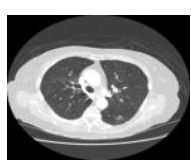

Input image

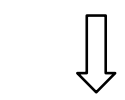

Image in FT domain

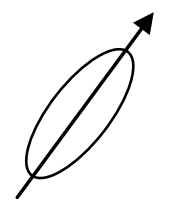

a curvelet at scale $\mathrm{s}$ And orientation $n$

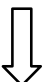

Curvelet in FT domain

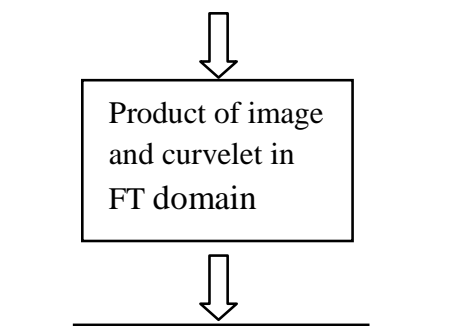

Wedge wrapping

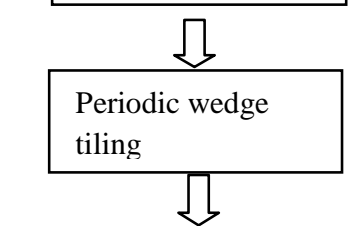

Curvelet Coefficient at scale $\mathrm{s}$ and orientation $\mathrm{n}$

Fig.1 Implementation of fast discrete curvelet transforms Once curvelet coefficients are computed, and then mean and standard deviation as the texture feature for the curvelet is computed.

\subsection{Neural Network}

Neural networks (ANN), commonly referred to as neural networks are the computational models motivated by human brain that are capable of machine learning and pattern recognition. The inherent parallel architecture and the fault tolerant nature of the ANN is mostly used to solve the variety of problems of imaging field, like in segmentation, feature extraction, classification and clustering. A neural network is defined as in [8]

"A neural network is massively parallel and distributed processor made up of simple processing units, which have natural propensity for storing experimental knowledge and making it available for future use" .It resembles brain in two aspects:-

1.) Knowledge is acquired by the network through the learning process.
2.) Interneuron connection strength known as synaptic weight, one used to store this acquired knowledge. Artificial neural network consist one input layer, one and more hidden layer and one output layer. With a novel algorithm ANN is trained with some input data, and after training phase, samples are tested in the testing phase to get proficient results.

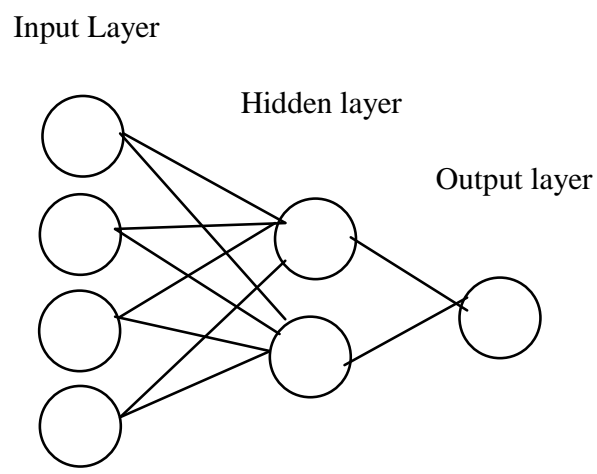

Fig.2 Feed forward Neural Network

In this proposed scheme we use these advantages of neural network for training and testing of lung cancer CT scan images.

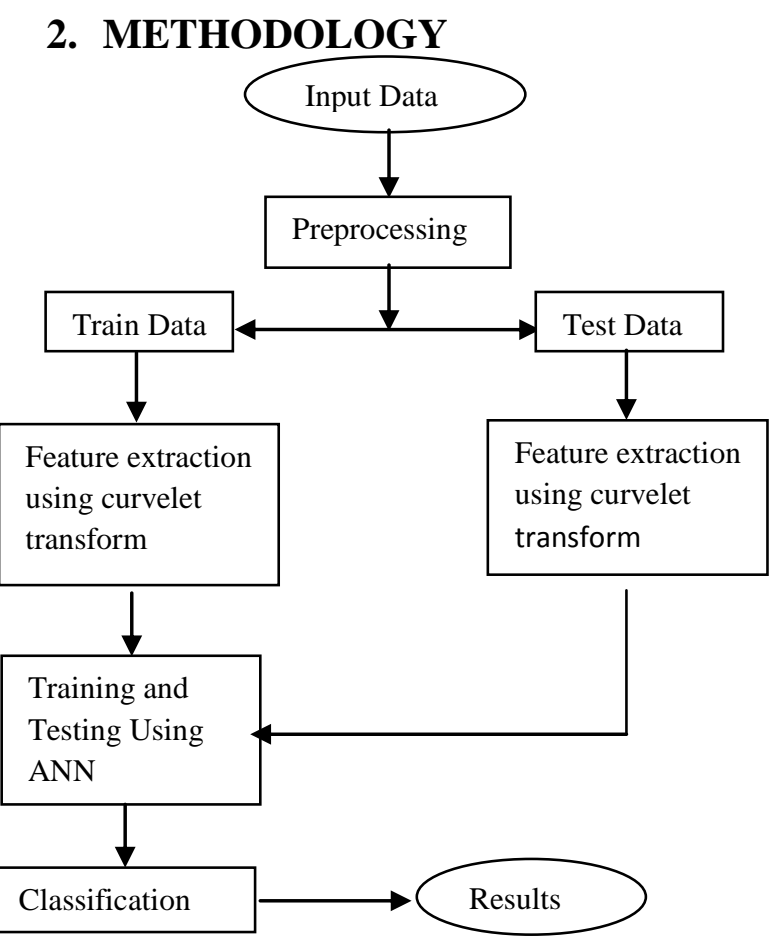

Fig.3 Flow chart of proposed work

The above flow chart shows our proposed work.

\subsubsection{Preprocessing:}

To enhance the contrast of images histogram equalization is used in numerous applications. The drawback of histogram equalization is that the image brightness is changed after apply this technique. To overcome the limitation of histogram equalization we used Contrast Limited Adaptive Histogram Equalization (CLAHE) technique to enhance CT scan images [9]. The CLAHE algorithm partitions the images into contextual regions and applies the histogram equalization to each one. This evens out the distribution of used grey values and thus makes hidden features of the image more visible. 


\subsubsection{Feature Extraction:}

After preprocessing feature of CT scan images are extracted using curvelet transform. Then mean and standard deviation are computed by using equ (5) and equ (6)

$$
\begin{aligned}
\mu & =\frac{\sum_{\mathrm{i}=1}^{\mathrm{i}=\mathrm{n}} \mathrm{Xi}}{\mathrm{n}} \\
\sigma & =\sqrt{1 /(\mathrm{n}-1) \sum_{\mathrm{i}=1}^{\mathrm{i}=\mathrm{n}}(\mathrm{Xi}-\mu)^{\wedge}} 2
\end{aligned}
$$

Here $X_{i}$ is the no of observed value, $n$ is total no observation, $\mu$ is mean and $\sigma$ is std.

\subsubsection{Training:}

Extracted features are used to train the neural network, positive samples (cancerous CT scan) are trained by 1 and negative samples (Non cancerous CT scan) by 0 . So, that this trained neural network will give the value close to target output 1 for image that contains lung nodule and for other it will give the value close to target output 0 .

\subsection{4: Classification:}

After training stage we classify the cancerous and non cancerous CT scan images. At the end we get trained network, if we test any positive and negative samples it give proficient results.

\subsubsection{Results:}

In this proposed system $90 \mathrm{CT}$ scans are used for training and $30 \mathrm{CT}$ scans for testing. Out of $90 \mathrm{CT}$ scans 45 are the cancerous which are trained by 1 and other 45 are the non cancerous, trained by 0 .

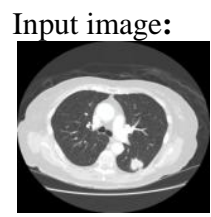

Fig.4 Input Image

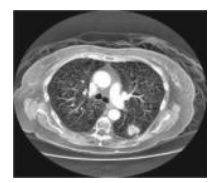

Fig.5 CLAHE Image
Figure 4 and Figure 5 shows the result of preprocessing step, in which CLAHE is used to improve the contrast. Then feature is extracted and these features are fed into the neural network for training. The performance of classification algorithm is evaluated by computing the percentages of Sensitivity (SE), Specificity (SP) and Accuracy (AC), the respective definition are as follows:

$$
\begin{aligned}
\mathrm{SE} & =\mathrm{TP} /(\mathrm{TP}+\mathrm{FN}) * 100 \\
\mathrm{SP} & =\mathrm{TN} /(\mathrm{TN}+\mathrm{FP}) * 100 \\
\mathrm{AC} & =(\mathrm{TP}+\mathrm{TN}) /(\mathrm{TP}+\mathrm{TN}+\mathrm{FP}+\mathrm{FN}) * 100
\end{aligned}
$$

Where TP is the number of true positives, TN is the number of true negatives, FN is the number of false negatives, and FP is the number of false positives, are defined as:

TP: Predicts cancerous as cancerous.

TN: Predicts noncancerous as noncancerous

FN: Predicts cancerous as noncancerous.

FP: Predicts noncancerous as cancerous.

Table 1: Results after testing the network

\begin{tabular}{|c|c|c|c|}
\hline $\begin{array}{c}\text { No. of } \\
\text { Images }\end{array}$ & Sensitivity & Specificity & Accuracy \\
\hline 30 & $86.66 \%$ & $93.33 \%$ & $90.00 \%$ \\
\hline
\end{tabular}

\section{CONCLUSION}

Lung cancer is the most dangerous diseases, so early detection of this diseases is necessary. But the detection of lung cancer is most difficult task. From the literature review many techniques are used for the detection of lung cancer but some limitations also exists. Our proposed method follows an approach in which first step is feature extraction, and then these features are used to train and test the neural network. Curvelet transform is used to extract the feature of CT scan images. From the results, the proposed technique successfully detects the lung cancer from CT scan images. Our proposed method gives $90 \%$ accuracy. If it is detected correctly in early stages then it increase the key of survival. In future this technique can be used in the detection of brain tumor.

\section{REFERENCES}

[1] Devaki, K., MuraliBhaskaran V.," Study of computed tomography images of the lungs: A survey" in "Recent Trends in Information Technology (ICRTIT) 2011 International Conference", OIEEE, doi 10.1109/ICRTIT.2011.5972308

[2] Patil, S.A. Udupi, V.R. ; Kane, C.D. ;Wasif, A.I. ; Desai, J.V. ; Jadhav, A.N.," Geometrical and texture features estimation of lung cancer and TB images using chest Xray database "in" Biomedical and Pharmaceutical Engineering, 2009. ICBPE' 09. International Conference"CIEEE,doi:10.1109/ICBPE.2009.5384113

[3] Yang Yu, Hong Zhao," A Texture-based Morphologic Enhancement Filter in Two-dimensional Thoracic CT scans" in" Networking, Sensing and Control, 2006 ICNSC '06. Proceedings of the 2006 IEEE International Conference" CIEEE, doi: 10.1109/ICNSC.2006.1673258

[4] Takemura, S., Xianhua Han; Yen-wei Chen; Ito, K. Nishikwa, I.; Ito, M. "Enhancement and detection of lung nodules with Multiscale filters in CT images " in" Intelligent Information Hiding and Multimedia Signal Processing, 2008. IIHMSP '08 International Conference", CIEEE,doi:10.1109/IIHMSP.2008.30

[5] E.J. Candes, D.L. Donoho, Curvelets, multi-resolution representation, and scaling laws, Wavelet Applications in Signal and Image Processing VIII, vol. 4119-01, SPIE, 2000

[6] Guesmi, H.; Trichili, H.; Alimi, A.M.; Solaiman, B., "Curvelet transform-based features extraction for fingerprint identification" in "Biometrics Special Interest Group (BIOSIG), 2012 BIOSIG - Proceedings of the International Conference" CIEEE

[7] Sumana, I.J. ; Islam, M.M. ; Dengsheng Zhang ; Guojun $\mathrm{Lu}$, "Content based image retrieval using curvelet transform" in" Multimedia Signal Processing, 2008 IEEE 10th Workshop" OIEEE, doi: 10.1109/MMSP.2008.4665041

[8] Haykin, Simon. Neural networks: a comprehensive foundation. Prentice Hall PTR, 1994.

[9] Ashwin, S., Kumar, S.A., Ramesh, J., Gunavathi, K., "Efficient and reliable lung nodule detection using a neural network based computer aided diagnosis system", in "Emerging Trends in Electrical Engineering and Energy Management (ICETEEEM), 2012 International Conference", $\quad$ CIEEE, 10.1109/ICETEEEM.2012.6494454. 